The effect of changes of surface curvature at the focus of an astronomical object glass

This content has been downloaded from IOPscience. Please scroll down to see the full text. 1922 Trans. Opt. Soc. 23241

(http://iopscience.iop.org/1475-4878/23/4/302)

View the table of contents for this issue, or go to the journal homepage for more

Download details:

IP Address: 130.237.165.40

This content was downloaded on 20/08/2015 at $16: 54$

Please note that terms and conditions apply. 


\title{
THE EFFECT OF CHANGES OF SURFACE CURVATURE AT THE FOCUS OF AN ASTRONOMICAL OBJECT GLASS
}

\author{
BY E. WILFRED TAYLOR \\ (Messrs T. Cooke \& Sons, Ltd., York) \\ MS. received, 27th Fanuary, 1922. Read and discussed, 6th April, r922.
}

$A B S T R A C T$. The correct balancing of the components of a large object glass is necessarily a lengthy and difficult process, and the effect, at the focus, of a similar alteration of curvature at each of the four surfaces, is different. If the effect of an alteration at each of the surfaces is known, the one most suitable may be chosen, having regard to the amount and nature of the aberration it is necessary to overcome.

FOR a large object glass the curves are accurately computed to eliminate spherical aberration and to bring the $\mathrm{C}$ and $\mathrm{F}$ rays to a common focus; thus in the case of an object glass of 6 -inches aperture the constants of the two discs are

$$
\begin{aligned}
& \text { Ordinary crown, } \mu_{D}=\mathrm{I} \cdot 5 \mathrm{I} 66 \text { and } \begin{array}{l}
\mu_{D}-\mathrm{I} \\
\mu_{C}-\mu_{F}
\end{array}=60.56273 \text {, } \\
& \text { Ordinary flint, } \mu_{D}=\mathrm{I} \cdot 6 \mathrm{I} 32 \text { and } \begin{array}{l}
\mu_{D}-\mathrm{I} \\
\mu_{C}-\mu_{F^{*}}
\end{array}=36.9 \mathrm{I} 732 .
\end{aligned}
$$

Taking the radius of curvature of the fourth surface (the surface through which the light passes last) as - I 80 inches, the curves come out as follows:

$$
\begin{aligned}
& \text { Crown lens }+29^{\circ} 1605^{\prime \prime} \text { and }+48 \cdot 2695^{\prime \prime} \text {, thickness } 5^{\prime \prime} \text {. } \\
& \text { Flint lens }-43 \cdot 9847^{\prime \prime} \text { and }-180^{\prime \prime} \text {, thickness } 40^{\prime \prime} \text {. }
\end{aligned}
$$

With an air gap of 10 in. between the two lenses, the marginal rays cut the axis .0043 in. farther from the back surface than do the axial rays. The object glass is therefore very slightly over-corrected for spherical aberration. The combined focal length is about 90 ins.

If an object glass was made exactly to these curves there would be little residual aberration to balance, but in practice the nearest tools will have to be used and when the object glass is first examined as a whole, it will show marked over- or undercorrection-this must be rectified by suitable alteration to the surfaces. To make a new set of tools is not usually worth while as the next discs will probably have different optical properties or a different focal length may be required for the object glass.

The deep surface of the negative lens is first figured until it is as perfect as possible when examined by back reflection, and the shallow surface is made deeper or shallower until the colour correction is approximately correct. (If the O.G. as a whole is under-corrected for aberration the colour should also be slightly undercorrected and vice versa.) Any irregularity of figure or any spherical aberration 
must now be assumed to be due to the positive element, where it must be corrected, but where it cannot readily be detected by direct examination as in the case of the negative surface.

At this stage the object glass will show more or less spherical aberration, depending on the departure from the theoretical curves, and this must be corrected by suitably altering one or other of the positive surfaces; that is by deepening or shallowing the surfaces, as the case may be. The new curve may not be truly spherical but will not in general depart appreciably from a spherical curve. These alterations to the crown lens will have a slight effect on the colour corrections. The colour correction can be finally perfected without any appreciable effect on the spherical aberration.

At this stage it is necessary to decide which of the two surfaces of the crown lens to select for alteration. In practice it is found that the inside surface of the crown lens exercises, for a corresponding change of curvature, a greater effect on the spherical aberration (and in consequence a smaller effect on the colour) than does the deep outside surface. At this stage one feels the desirability of knowing definitely what is the effect at the focus of a given change in power at any one of the surfaces, with a view to choosing the one most suitable for the purpose in view.

In polishing, the maximum alteration to a curve will be about half the spacing between one tool and the next and $\frac{I}{r} \pm-\frac{I}{2000}$ may be taken to represent the limits likely to occur in practice, where $r=$ radius of curvature. The limits for each surface will then be :

(a)

Maximum

Correct

Minimum (b)

$$
\begin{aligned}
& +49.46327^{\prime \prime} \\
& +48.2695^{\prime \prime}
\end{aligned}
$$$$
+47^{\circ} \text { ' } 3 \text { I } 97^{\prime \prime}
$$

(c)

$$
\begin{array}{ll}
-44.973^{8} 3^{\prime \prime} & -197 \cdot 8022^{\prime \prime} \\
-43.98466^{\prime \prime} & -180^{\prime \prime} \\
-43.03815^{\prime \prime} & -165^{\circ} \cdot 377^{\prime \prime}
\end{array}
$$

In order to determine the effect, at the focus, of an alteration in power of each surface, from a maximum to a correct value and from a correct value to a minimum value, the other three surfaces were left unchanged at their correct values. The linear value of the spherical aberration was worked out trigonometrically, the slight overcorrection of $.00434^{\prime \prime}$ in the case of the calculated values being allowed for in each case.

Denoting over-correction by a + sign and under-correction by a - sign, and taking the four surfaces in the order that the light traverses them, we get:

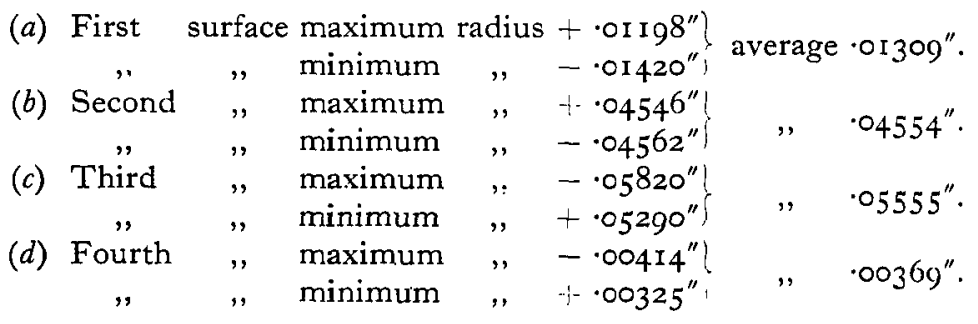


Taking the effect of an alteration at the fourth surface as unity the figures expressing for the four surfaces the average amount of the spherical aberration produced at the focus are $3.54,12.34,15.06$, and $x$. It will be seen that to produce a given alteration at the focus, a much greater alteration will be required at surface (a) than at surface $(b)$ and very much more at surface $(d)$ than at surface $(c)$. It will also be seen that the effect on the spherical aberration of an alteration to surface (d) is only negligible when the alteration is slight.

From these figures we may deduce the best procedure, in the correcting of a large object glass, as follows:

(I) Correct the figure of the third surface, making it as perfect as possible.

(2) Alter the radius of the back surface until the o.G. as a whole is about correct for colour. If the object glass is under-corrected for aberration the colour correction should also err on the underside. Obtain a perfect figure.

(3) Approximately correct the spherical aberration on the second surface of the crown, where the effect is comparatively great. Alteration at this surface has the least effect on the colour for a given change in the spherical aberration, the proportional alterations to colour for the four surfaces for the same alteration of spherical aberration being $-3 \cdot 48,1,1 \cdot 34$, and $20 \cdot 24$, where that of the second surface is taken as unity.

(4) Obtain final perfection by alteration to the first surface.

(5) If necessary, re-correct the colour by alteration to the fourth surface and again obtain a perfect figure. The correction for spherical aberration will be unaffected as the alteration will be slight.

In this paper it has been assumed that the focal length can vary and it is improbable that the final focal length will be $90^{\prime \prime}$ as it was in the case of the calculated curves. Generally a change of one or two inches is immaterial but if an object glass is required to have a certain focal length, within small limits the procedure will be different and it may have finally to be obtained by slightly modifying the colour correction.

It is an interesting fact that, although it is the aim in designing an object glass of this type to bring the $\mathrm{C}$ and $\mathrm{F}$ rays to a common focus, this cannot be perfectly accomplished. If, for instance, the axial $\mathrm{C}$ and $\mathrm{F}$ rays meet at a common focus, then those that pass through the margin of the lens cannot do so. This is due to the fact that if the $\mathrm{D}$ rays are perfectly corrected for spherical aberration, the $\mathrm{C}$ rays will be under-corrected and the $F$ rays over-corrected. Probably the best colour correction possible consists in bringing together the circles of least confusion for the $\mathrm{C}$ and $\mathrm{F}$ rays.

In the case of the object glass considered above, when the $\mathrm{D}$ rays are perfectly corrected for spherical aberration, the linear under-correction for the $\mathrm{C}$ rays is $-.00424^{\prime}$ and for the $\mathrm{F}$ rays the linear over-correction is + .01606". 


\section{DISCUSSION}

Mr T. Smith (communicated): This paper is particularly interesting in that it gives the procedure in correcting objectives which the very extended experience of Messrs T. Cooke \& Sons has shown to be most satisfactory. If numerical evaluation of the effects of known curvature alterations is not required, but it is merely desired to find which surface should preferably be altered, a decision can be reached on quite general grounds. The alteration of a single surface may be regarded as (I) a change in the power of a component without a change in the shape, together with (2) a change in the shape without any change in the power. This way of regarding the change is equivalent to resolving any displacement of a point into two components in assigned directions; the point of intersection of the two surfaces in any diametral section when the curvature of one surface is altered moves along the other surface; (I) corresponds to a displacement towards or away from the axis, and (2) a displacement parallel to the axis. The effects of (I) are the same whichever surface is altered, but the effects of (2) are dependent in sign upon the surface selected. The chromatic aberration is determined solely by (I), but spherical aberration is dependent upon both, and consequently the greatest change in spherical aberration for a given change in chromatic aberration is obtained by altering the side for which the two contributions to the change in spherical aberration agree in sign. The sign of the change due to $(\mathrm{I})$ is evident, and the change in spherical aberration under (2) has the same sign as the power of the component lens when the lens is bent further away from the form for which the aberration is a minimum. In the present case if the colour is over-corrected the power of the crown lens must be increased, and the contribution to spherical aberration under ( $\mathrm{r}$ ) is positive. To obtain a positive contribution from (2), the crown lens must be made less near the "crossed" form, that is to say, the second surface must be altered. If the colour is under-corrected, all changes must have their signs reversed, and the surface to be altered is not affected.

$\mathrm{Mr}$ S. J. Underhill thought that the procedure outlined by the author was applicable only to lenses having an aperture ratio of less than F/9, and that lenses having greater aperture ratios must have their surfaces deformed from the spherical shape to correct for spherical aberration. This process can be exactly performed by utilising a special instrument*, in which both the amount of glass to be removed and the zone upon which the removal is to be made are seen at the same time. The correction can then be quickly and accurately performed by a skilled worker.

$\mathrm{Mr} \mathrm{H}$. S. Ryland said that some years ago Mr Max Zwillinger had constructed objectives which fulfilled the sine conditions and were free from spherical aberrations. He obtained good results by figuring the back surface, but the process was very expensive.

* Trans. Opt. Soc. 22 (1920-21), 174. 
Author's reply (communicated): General methods, such as the one given by $\mathrm{Mr}$ Smith for deducing the effect at the focus of an object glass due to changes at the surfaces, are extremely useful. It is chiefly in the case of large surfaces, where the cost of figuring is high, that general considerations are not sufficient.

The aperture ratios, for which these results were intended to apply, vary between F/ro and F/r 5 .

In reply to $\mathrm{Mr}$ Ryland, I should like to say that this firm has frequently made object glasses that both fulfilled the sine condition and were free from spherical aberration. These are usually supplied with Transit Instruments where a large field is required. 